\title{
Sintomas depressivos e fatores associados entre idosos residentes em uma comunidade no norte de Minas Gerais, Brasil
}

Depressive symptoms and associated factors among elders dwelling

in a community in the North of Minas Gerais state, Brazil

Ernesto José Hoffmann', Fabio Ribeiro², Jussara Martins Farnese³ ${ }^{3}$ Estefânia Wanderley Barbosa Lima

\section{RESUMO}

Objetivo: Verificar a prevalência de sintomas depressivos de uma população idosa residente em uma comunidade de Montes Claros, Minas Gerais, Brasil, e avaliar sua associação com aspectos biopsicossociais e capacidade funcional. Métodos: Estudo transversal, observacional e de base populacional. Foi avaliada a totalidade dos idosos residentes na área de abrangência de uma Equipe de Saúde da Família, sendo eles entrevistados em seus domicílios. Foi utilizado questionário adaptado do Brazil Old Age Schedule (BOAS) para coletar os dados biopsicossociais. Para avaliar sintomas depressivos, foi aplicada a Escala de Depressão Geriátrica abreviada (EDG-15). A escala de Katz foi utilizada para avaliar as atividades básicas de vida diária (ABVD) e a escala de Pfeffer, para atividades instrumentais de vida diária (AIVD). As associações foram verificadas mediante submissão dos dados à análise bivariada e multivariada. Resultados: A prevalência de sintomatologia depressiva foi de 20,9\%. Apresentaram

\section{Palavras-chave}

Atenção primária à saúde, idoso, depressão, atividades cotidianas. associação significativa com sintomas depressivos: dificuldade para dormir ( $R P=2,04 ; \mathrm{p}=$ $0,002)$ e dependência para AIVD ( $R P=3,22 ; p<0,001)$. Conclusão: Há alta prevalência de sintomas depressivos entre idosos no âmbito comunitário, sendo mais frequentes entre idosos com dificuldade para dormir e com dependência funcional para AIVD.

\section{ABSTRACT}

Objective: To verify the prevalence of depressive symptoms of an elder population dwelling in a community in Montes Claros, Minas Gerais, Brazil, and evaluate the association of them with biopsychosocial aspects and functional capacity. Methods: Transversal, observational, and population-based study. All of the elders living in the coverage area of a Family Health Team, and the interviews were made in their dwellings. An adaptation of the Brazil Old Age Schedule (BOAS) questionnaire was used to collect the biopsychosocial data. The abbreviated Geriatric Depression Scale (GDS-15) was used to evaluate depressive symptoms. The Katz index was used to evaluate the basic activities of daily living (BADL); the Pfeffer index to evaluate instrumental activities of daily living (IADL). The associations were verified by submitting data to bivariate and multivariate analysis.

1 Universidade Estadual de Montes Claros (Unimontes), Faculdade de Medicina, Centro de Ciências Biológicas e da Saúde, Departamento de Saúde Mental e Coletiva. 


\section{Keywords}

Primary health care, aged, depression, activities of daily living.
Results: The prevalence of depressive symptoms was of 20.9\%. Depressive symptoms were significative associated with difficulty to sleep $(P R=2.04 ; p=0.002)$ and dependence for IADL $(P R=3.22 ; P<0.001)$. Conclusion: There is a high prevalence of depressive symptoms among elders in the community, being more frequent among elders with difficulty to sleep and functional dependence for IADL.

\section{INTRODUÇÃO}

Dentre as enfermidades crônicas passíveis de afetar pacientes idosos, os problemas do campo da saúde mental representam parcela importante. Entre eles, os distúrbios afetivos com alterações do humor são os principais ${ }^{1}$, com especial destaque para aqueles apresentando sintomatologia depressiva.

Sintomas depressivos têm alta prevalência na população idosa, porém esta sofre importante variação dependendo da escala utilizada para avaliar tais sintomas e a região onde vivem os indivíduos avaliados ${ }^{2}$. No entanto, a maioria dos estudos de prevalência apresenta resultado superior a 10\% para transtornos depressivos em idosos requerendo tratamento². No Brasil, dois estudos realizados em diferentes regiões com idosos residentes na comunidade apresentaram prevalência de sintomatologia depressiva em torno de $20 \%$.

Apesar de prevalentes, sintomas depressivos usualmente são pouco investigados ${ }^{5}$ e subdiagnosticados ${ }^{6}$ na população idosa. Demência, doenças físicas, perda da independência e suicídio são muito mais comuns na velhice, corroborando para maior prevalência de depressão nessa população ${ }^{7}$. Em contrapartida, o não tratamento da depressão no idoso pode piorar o prognóstico de enfermidades físicas, causar alterações cognitivas e aumentar o índice de mortalidade desses indivíduos ${ }^{8}$, levando a maior sofrimento do paciente e de sua família e a maiores gastos com cuidados de saúde.

É importante, também, mencionar alguns fatores descritos como associados à depressão na literatura. Uma metanálise de 20 estudos longitudinais publicados em língua inglesa e francesa demonstrou como importantes fatores de risco para depressão entre idosos comunitários o luto, distúrbios do sono, incapacidade, depressão prévia e sexo feminino ${ }^{9}$. No Brasil, um estudo transversal avaliando mais de 7 mil indivíduos idosos residentes na comunidade evidenciou baixa renda e piores condições sociais, de saúde e funcionais como fatores associados à depressão 3 .

Dentre esses fatores associados, cabe ressaltar a incapacidade funcional, ou seja, a dependência desse grupo de pacientes do auxílio de outros para a realização de atividades de vida diária (AVD). Tal incapacidade pode afetar consideravelmente a qualidade de vida deles e ter influência nos cuidados futuros ${ }^{10}$. Além disso, a incapacidade funcional encontra-se associada de forma bidirecional com sintomas depressivos, podendo ser apontada na literatura tanto como fator de risco ${ }^{9}$ como fator resultante dele $e^{11,12}$.
Por todos esses motivos, esforços são justificados no sentido de identificar casos suspeitos de depressão, bem como realizar uma correta avaliação da capacidade funcional desses indivíduos, em especial no âmbito da atenção primária à saúde, uma vez que é no nível comunitário em que se encontra habitando a maior parcela da população idosa e se oferece a maior parte do atendimento a esses indivíduos ${ }^{13}$.

O presente trabalho objetiva identificar a prevalência de sintomas depressivos e avaliar fatores associados em uma população idosa residente na área de abrangência de uma equipe de Estratégia de Saúde da Família (ESF) na cidade de Montes Claros, MG.

\section{MÉTODOS}

Foi realizado estudo transversal, observacional e de base populacional na cidade de Montes Claros, norte de Minas Gerais, uma das regiões mais pobres e com piores índices de desenvolvimento desse estado, de junho a agosto de 2009. Objetivou-se avaliar todos os idosos cadastrados pela ESF Cintra II, desse município, compreendendo um universo de 314 pessoas. Os critérios de inclusão foram: indivíduos com idade igual ou superior a 60 anos, residentes na área de abrangência dessa unidade de saúde, que não possuam déficit auditivo ou cognitivo impedindo a compreensão das perguntas a lhes serem dirigidas, tendo recebido avaliação clínica prévia pelo médico da ESF para esse critério. Tal médico recebeu capacitação do Centro de Referência em Assistência à Saúde do Idoso (CRASI) de Montes Claros para tais avaliações. Especificamente para a cognição, foi utilizado o Miniexame do Estado Mental ${ }^{14}$ como método avaliativo. Foram excluídos os indivíduos não encontrados após três tentativas em dias e horários diferentes. As entrevistas com os idosos foram realizadas em seus respectivos domicílios por 10 alunos de medicina previamente treinados para a aplicação dos instrumentos:

- Questionário para avaliação de aspectos biopsicossociais, adaptado do Brazil Old Age Schedule (BOAS), desenvolvido na Inglaterra e modificado posteriormente por pesquisadores do Rio de Janeiro ${ }^{15}$. O questionário avaliou: sexo, idade, escolaridade, estado conjugal, número de pessoas morando com o idoso, forma de sustento de vida, renda, padrão de alimentação, presença de problema de saúde, uso de medicamentos, perda de parente ou companheiro nos últimos meses e dificuldade para dormir. 
- Escala de Depressão Geriátrica abreviada de 15 questões (EDG-15) ${ }^{16}$. Foi utilizada para avaliar sintomas depressivos. Cada pergunta respondida de forma a sugerir tais sintomas somou um ponto. Idosos com escores maiores que 5 foram considerados com sintomatologia depressiva presente.

- Escala de Katz" ${ }^{17}$. Usada para avaliar atividades básicas de vida diária (ABVD): banhar-se, vestir-se, realizar sua higiene pessoal, transferir-se, alimentar-se e controlar esfíncteres. Somouse um ponto para cada atividade que o idoso conseguia realizar satisfatoriamente. Os idosos com pontuação de 5 a 6 foram considerados independentes para ABVD; de 3 a 4, com dependência parcial; e de 0 a 2, com dependência importante.

- Escala de Pfeffer ${ }^{18}$. Foi usada para avaliar as atividades instrumentais de vida diária (AIVD): cuidar do próprio dinheiro, fazer compras sozinho, esquentar água e apagar o fogo, preparar comida, manter-se a par dos acontecimentos da vizinhança, prestar atenção, entender e discutir notícias, lembrar de compromissos e acontecimentos familiares, cuidar de seus medicamentos, andar pela vizinhança e voltar para casa, cumprimentar amigos, ficar sozinho em casa sem problemas. Para cada um dos itens, o idoso não somava pontos se fosse capaz de realizá-los, somava 1 ponto se tivesse dificuldade para fazê-los, 2 pontos se necessitasse de ajuda e 3 pontos se fosse incapaz de cumpri-los satisfatoriamente. Os indivíduos com escore final de 5 pontos ou menos foram classificados como independentes funcionais e aqueles com 6 ou mais, como dependentes.

$O$ treinamento foi realizado em dois momentos, englobando demonstração expositiva dos instrumentos, explicação pergunta a pergunta de cada um dos instrumentos e espaço para elucidação de dúvidas. Foi realizado, em seguida, estudo piloto visando à aplicabilidade e à viabilidade dos instrumentos em uso. Estes foram avaliados duas vezes para testar a compreensão das questões pelos idosos. O estudo piloto foi realizado com indivíduos cadastrados em outra ESF presente no mesmo bairro. Para tanto, 10 domicílios que possuíam indivíduos com 60 anos ou mais como residentes foram selecionados de forma aleatória. Logo após o estudo piloto, foi iniciada a coleta de dados.

O projeto desta pesquisa foi submetido ao Comitê de Ética em Pesquisa envolvendo Seres Humanos da Universidade Estadual de Montes Claros (Unimontes), tendo sido avaliado e aprovado mediante o parecer do processo de número 1.186/08 e conduzido dentro dos preceitos éticos vigentes. Para a realização da pesquisa, foi utilizado um Termo de Consentimento Livre e Esclarecido. Todos os sujeitos abordados foram inicialmente informados quanto à identificação dos entrevistadores, aos objetivos da pesquisa, a não obrigatoriedade de participação e à possibilidade de abandono do projeto a qualquer momento. Os problemas de saúde dos idosos avaliados foram considerados pelo acesso a atendimento na própria ESF. Aqueles identificados com sintomas depressivos foram encaminhados para o CRASI para avaliação clínica complementar de acordo com os critérios do DSM-IV19. Os idosos que, por fim, obtiveram diagnóstico de depressão receberam tratamento medicamentoso e acompanhamento médico e psicológico específicos.

A análise dos dados foi realizada empregando o software estatístico SPSS ${ }^{\oplus}$, versão 15.0 para Windows ${ }^{\oplus}$. Inicialmente, foi realizada análise descritiva dos dados. Em seguida, investigou-se a existência de associação estatística entre a variável dependente presença de sintomas depressivos e as variáveis independentes, relativas à capacidade funcional e aspectos biopsicossociais. Para tanto, foram conduzidas análises bivariadas (teste do qui-quadrado de Pearson) e análises multivariadas, por meio de regressão de Poisson, e estimadas as razões de prevalência com seus respectivos intervalos de confiança de $95 \%$ e valores de p. As variáveis que apresentaram níveis descritivos (valores de p) menores que 0,25 na análise bivariada foram incluídas na análise multivariada seguindo a ordem decrescente do nível descritivo ${ }^{20}$. O modelo final foi composto pelos fatores que permaneceram associados aos sintomas depressivos ao nível de 0,05 $(p<0,05)$.

\section{RESULTADOS}

Foi avaliado um total de 258 idosos cadastrados pela ESF Cintra II. Dos 314 indivíduos inicialmente pertencentes ao universo do estudo, 30 não foram encontrados em seus domicílios após três tentativas, 10 mudaram-se, 10 negaram-se a participar e 6 faleceram durante a coleta de dados da pesquisa. As idades variaram entre 60 e 98 anos, com média de 71,1 anos ( $D P= \pm 8,08)$. Dos idosos avaliados, 168 foram do sexo feminino, representando quase dois terços da população estudada $(65,1 \%)$. A maior parte dos entrevistados demonstrou o seguinte perfil: idade entre 60 e 80 anos (83,7\%); primário completo (45,3\%); casados ou com relacionamento estável (49,2\%); morando com 2 até 4 pessoas (48,4\%); aposentados $(65,9 \%)$; renda familiar até 1 salário-mínimo (35,2\%); relatando alimentar-se bem (85,3\%); apresentando algum problema de saúde $(86,0 \%)$ e usando 2 até 3 medicamentos (46,9\%). A maioria dos idosos também negou a perda de parente ou companheiro nos últimos meses $(69,9 \%)$ e que apresenta dificuldade para dormir (64,3\%). A distribuição das variáveis relativas aos aspectos biopsicossociais encontra-se na tabela 1.

Os sintomas depressivos foram observados em 54 idosos (20,9\%). Com relação às ABVD, a grande maioria dos idosos $(96,9 \%)$ se apresentou independente. Já para as AIVD, um número expressivo demonstrou dependência funcional: 34 sujeitos (13,2\%) (Tabela 2).

Na análise bivariada, os sintomas depressivos foram significativamente associados com não estar se alimentando bem $(p=0,001)$, fazer uso de mais de 3 medicamentos $(p=0,004)$, ter perdido parente ou companheiro nos últimos meses $(p=0,017)$, apresentar dificuldade para dormir $(p=$ $0,002)$, dependência parcial para ABVD $(p<0,001)$ e dependência para AIVD $(p<0,001)$ (Tabela 3). 
Tabela 1. Distribuição das variáveis biopsicossociais (idosos cadastrados ESF Cintra II, Montes Claros, MG, 2009)

\begin{tabular}{|c|c|c|}
\hline Variáveis & $\mathrm{n}$ & $\%$ \\
\hline \multicolumn{3}{|l|}{ Gênero } \\
\hline Masculino & 90 & 34,9 \\
\hline Feminino & 168 & 65,1 \\
\hline \multicolumn{3}{|l|}{ Idade } \\
\hline De 60 a 80 anos & 216 & 83,7 \\
\hline Maior de 80 anos & 42 & 16,3 \\
\hline \multicolumn{3}{|l|}{ Escolaridade completa } \\
\hline $2^{\circ} \mathrm{grau}$ e superior & 32 & 12,4 \\
\hline $1^{\circ} \mathrm{grau}$ & 43 & 16,7 \\
\hline Primário & 117 & 45,3 \\
\hline Nenhuma & 66 & 25,6 \\
\hline \multicolumn{3}{|l|}{ Estado conjugal } \\
\hline Casado/relação estável & 127 & 49,2 \\
\hline Separado & 17 & 6,6 \\
\hline Viúvo & 94 & 36,4 \\
\hline Nunca casou & 20 & 7,8 \\
\hline \multicolumn{3}{|l|}{ Pessoas morando com o idoso } \\
\hline 5 pessoas ou mais & 51 & 19,8 \\
\hline 2 até 4 pessoas & 125 & 48,4 \\
\hline 1 pessoa & 58 & 22,5 \\
\hline Mora só & 24 & 9,3 \\
\hline \multicolumn{3}{|l|}{ Sustento de vida } \\
\hline Trabalho & 20 & 7,8 \\
\hline Aposentadoria & 170 & 65,9 \\
\hline $\begin{array}{l}\text { Pensão/ajuda esposo(a), ajuda de parentes/amigos, } \\
\text { aluguéis/investimentos, outros }\end{array}$ & 68 & 26,4 \\
\hline \multicolumn{3}{|l|}{ Renda familiar } \\
\hline > 3 salários-mínimos & 47 & $21,5^{*}$ \\
\hline$>2$ a 3 salários-mínimos & 32 & $14,6^{*}$ \\
\hline > 1 a 2 salários-mínimos & 63 & $28,8^{*}$ \\
\hline Até 1 salário-mínimo & 77 & $35,2^{*}$ \\
\hline \multicolumn{3}{|l|}{ Alimenta-se bem } \\
\hline $\operatorname{sim}$ & 220 & 85,3 \\
\hline Não & 38 & 14,7 \\
\hline \multicolumn{3}{|l|}{ Problema de saúde } \\
\hline Não & 36 & 14,0 \\
\hline Sim & 222 & 86,0 \\
\hline \multicolumn{3}{|l|}{ Número de medicamentos em uso } \\
\hline 0 a 1 & 89 & 34,5 \\
\hline 2 a 3 & 121 & 46,9 \\
\hline$>3$ & 48 & 18,6 \\
\hline \multicolumn{3}{|l|}{ Perda de parente ou companheiro nos últimos meses } \\
\hline Não & 179 & $69,9^{*}$ \\
\hline Sim & 77 & $30,1^{*}$ \\
\hline \multicolumn{3}{|l|}{ Dificuldade para dormir } \\
\hline Não & 166 & 64,3 \\
\hline Sim & 92 & 35,7 \\
\hline
\end{tabular}

Tabela 2. Distribuição dos sintomas depressivos, ABVD e AIVD. Idosos cadastrados pela Equipe de Saúde de Família Cintra II, Montes Claros - MG, 2009

\begin{tabular}{lll}
\hline Variáveis & $\mathbf{n}$ & $\%$ \\
Sintomas depressivos & & \\
$\quad$ Ausentes & 204 & 79,1 \\
$\quad$ Presentes & 54 & 20,9 \\
$\begin{array}{l}\text { Atividades Básicas de Vida Diária } \\
\text { Independência }\end{array}$ & 250 & 96,9 \\
$\quad \begin{array}{l}\text { Dependência parcial } \\
\text { Dependência importante }\end{array}$ & 2 & 0,8 \\
Atividades Instrumentais de Vida Diária & 6 & 2,3 \\
$\quad$ Independência funcional & & \\
$\quad$ Dependência & 224 & 86,8 \\
\hline
\end{tabular}

Após a análise multivariada, permaneceu significativamente associado com sintomas depressivos apresentar dificuldade para dormir ( $R P=2,04)$ e dependência para AIVD (RP $=3,22)$ (Tabela 4).

\section{DISCUSSÃO}

O presente estudo permitiu avaliar, relativamente aos aspectos estudados, as condições de saúde atuais dos idosos residentes na área de abrangência da ESF Cintra II.

Com relação à população estudada, a maior prevalência de indivíduos do sexo feminino foi condizente com a tendência nacional, que demonstra uma feminização da população idosa ${ }^{21}$. Também se pode perceber tratar-se de uma população com baixo nível de escolaridade e baixa renda familiar, resultados esperados diante da realidade socioeconômica local e mais uma vez congruentes com a realidade brasileira ${ }^{21}$.

A prevalência de sintomas depressivos na população estudada foi de 20,9\%. Trabalhos recentes não são muito conclusivos com relação à prevalência desses sintomas em idosos em âmbito comunitário. Há grande variabilidade nos achados entre países², e mesmo em diferentes comunidades no Brasil. Estudo avaliando 1.563 idosos comunitários em São Paulo demonstrou prevalência de sintomas depressivos de $13 \% 22$. Já em estudo realizado em idosos em uma comunidade no nordeste do Brasil encontrou 25,5\% ${ }^{23}$. O resultado do presente trabalho apresenta prevalência semelhante ao de outro estudo também conduzido no estado de Minas Gerais, na cidade de Bambuí, que apontou 19,2\% da população idosa com sintomatologia depressiva presente ${ }^{4}$. As diferentes prevalências entre estudos talvez possam ser explicadas pelos métodos empregados, variabilidade no número de indivíduos estudados e diferentes pontos de corte relativos à idade como critério de inclusão, bem como a características peculiares de cada população. 
Tabela 3. Resultado da análise bivariada (qui-quadrado) - Associação entre sintomas depressivos e as variáveis independentes

\begin{tabular}{|c|c|c|c|c|}
\hline Variáveis & $\begin{array}{l}\text { Sintomas depressivos presentes } \\
\mathrm{n}(\%)\end{array}$ & $\begin{array}{l}\text { Sintomas depressivos ausentes } \\
\mathrm{n}(\%)\end{array}$ & $\operatorname{RP}(\mathbf{I C 9 5 \% )}$ & Valor $p$ \\
\hline \multicolumn{5}{|l|}{ Gênero } \\
\hline Masculino & $14(15,6)$ & $76(84,4)$ & 1,00 & \\
\hline Feminino & $40(23,8)$ & $128(76,2)$ & $1,53(0,88-2,66)$ & 0,131 \\
\hline \multicolumn{5}{|l|}{ Idade } \\
\hline De 60 a 80 anos & $41(19,0)$ & $175(81,0)$ & 1,00 & \\
\hline Maior de 80 anos & $13(31,0)$ & $29(69,0)$ & $1,63(0,96-2,77)$ & 0,070 \\
\hline \multicolumn{5}{|l|}{ Escolaridade completa } \\
\hline $2^{\circ}$ grau e superior & $6(18,8)$ & $26(81,2)$ & 1,00 & \\
\hline $1^{\circ} \mathrm{grau}$ & $3(7,0)$ & $40(93,0)$ & $0,372(0,10-1,38)$ & 0,139 \\
\hline Primário & $27(23,1)$ & $90(76,9)$ & $1,23(0,56-2,72)$ & 0,608 \\
\hline Nenhuma & $18(27,3)$ & $48(72,7)$ & $1,46(0,64-3,31)$ & 0,372 \\
\hline \multicolumn{5}{|l|}{ Estado conjugal } \\
\hline Casado/relação estável & $24(18,9)$ & $103(81,1)$ & 1,00 & \\
\hline Separado & $6(35,3)$ & $11(64,7)$ & $1,87(0,89-3,91)$ & 0,097 \\
\hline Viúvo & $21(22,3)$ & $73(77,7)$ & $0,79(0,26-2,39)$ & 0,682 \\
\hline Nunca casou & $3(15,0)$ & $17(85,0)$ & $1,18(0,70-1,99)$ & 0,529 \\
\hline \multicolumn{5}{|l|}{ Pessoas morando com o idoso } \\
\hline 5 pessoas ou mais & $7(13,7)$ & $44(86,3)$ & 1,00 & \\
\hline 2 até 4 pessoas & $25(20,0)$ & $100(80,0)$ & $1,46(0,67-3,15)$ & 0,339 \\
\hline 1 pessoa & $16(27,6)$ & $42(72,4)$ & $2,01(0,90-4,49)$ & 0,089 \\
\hline Mora só & $6(25,0)$ & $18(75,0)$ & $1,82(0,69-4,84)$ & 0,229 \\
\hline \multicolumn{5}{|l|}{ Sustento de vida } \\
\hline Trabalho & $2(10,0)$ & $18(90,0)$ & 1,00 & \\
\hline Aposentadoria & $37(21,8)$ & $133(78,2)$ & $2,18(0,57-8,36)$ & 0,257 \\
\hline $\begin{array}{l}\text { Pensão/ajuda esposo(a), ajuda de parentes/amigos, aluguéis/ } \\
\text { investimentos, outros }\end{array}$ & $15(22,1)$ & $53(77,9)$ & $2,21(0,55-8,84)$ & 0,264 \\
\hline \multicolumn{5}{|l|}{ Renda familiar } \\
\hline > 3 salários-mínimos & $5(10,6)^{*}$ & $42(89,4)^{*}$ & 1,00 & \\
\hline$>2$ até 3 salários-mínimos & $6(18,8)^{*}$ & $26(81,2)^{*}$ & $1,76(0,59-5,29)$ & 0,312 \\
\hline$>1$ até 2 salários-mínimos & $16(25,4)^{*}$ & $47(74,6)^{*}$ & $2,39(0,94-6,05)$ & 0,067 \\
\hline Até 1 salário-mínimo & $16(20,8)^{*}$ & $61(79,2)^{*}$ & $1,95(0,77-4,98)$ & 0,161 \\
\hline \multicolumn{5}{|l|}{ Alimenta-se bem } \\
\hline $\operatorname{sim}$ & $39(17,7)$ & $181(82,3)$ & 1,00 & \\
\hline Não & $15(39,5)$ & $23(60,5)$ & $2,23(1,37-3,62)$ & 0,001 \\
\hline \multicolumn{5}{|l|}{ Problema de saúde } \\
\hline Não & $3(8,3)$ & $33(91,7)$ & 1,00 & \\
\hline $\operatorname{Sim}$ & $51(23,0)$ & $171(77,0)$ & $2,76(0,91-8,36)$ & 0,073 \\
\hline \multicolumn{5}{|l|}{ Número de medicamentos em uso } \\
\hline 0a 1 & $11(12,4)$ & $78(87,6)$ & 1,00 & \\
\hline 2 a 3 & $27(22,3)$ & $94(77,7)$ & $1,81(0,95-3,44)$ & 0,073 \\
\hline$>3$ & $16(33,3)$ & $32(66,7)$ & $2,70(1,36-5,34)$ & 0,004 \\
\hline \multicolumn{5}{|l|}{ Perda de parente ou companheiro nos últimos meses } \\
\hline Não & $30(16,8)^{*}$ & $149(83,2)^{*}$ & 1,00 & \\
\hline Sim & $23(29,9)^{*}$ & $54(70,1)^{*}$ & $1,78(1,11-2,86)$ & 0,017 \\
\hline \multicolumn{5}{|l|}{ Dificuldade para dormir } \\
\hline Não & $25(15,1)$ & $141(84,9)$ & 1,00 & \\
\hline Sim & $29(31,5)$ & $63(68,5)$ & $2,09(1,31-3,35)$ & 0,002 \\
\hline \multicolumn{5}{|l|}{ Atividades básicas de vida diária } \\
\hline Independência & $49(19,6)$ & $201(80,4)$ & 1,00 & \\
\hline Dependência parcial & $1(50,0)$ & $1(50,0)$ & $2,56(0,62-10,43)$ & $<0,001$ \\
\hline Dependência importante & $4(66,7)$ & $2(33,3)$ & $3,40(1,83-6,32)$ & 0,193 \\
\hline \multicolumn{5}{|l|}{ Atividades instrumentais de vida diária } \\
\hline Independência funcional & $36(16,1)$ & $188(83,9)$ & 1,00 & \\
\hline Dependência & $18(52,9)$ & $16(47,1)$ & $3,29(2,13-5,09)$ & $<0,001$ \\
\hline
\end{tabular}

* Porcentagens válidas, excluindo-se as perdas. 
Tabela 4. Resultado da análise multivariada por meio da regressão de Poisson - Modelo final para sintomas depressivos

\begin{tabular}{lll}
\hline Variáveis & $\mathrm{RP}(\mathrm{IC95 \% )}$ & Valor p \\
\hline Atividades instrumentais de vida diária & & \\
Independência funcional & 1,00 & \\
Dependência & $3,22(2,08-5,00)$ & $<\mathbf{0 , 0 0 1}$ \\
Apresenta dificuldade para dormir & & \\
Não & 1,00 & \\
Sim & $2,04(1,30-3,21)$ & $\mathbf{0 , 0 0 2}$ \\
\hline
\end{tabular}

Fatores associados aos sintomas depressivos descritos na literatura, como baixa renda e piores condições sociais ${ }^{3}$, também podem ajudar a explicar tais diferenças. $O$ fato de o estudo realizado em São Paulo ter abordado uma população urbana e com níveis socioeconômicos diversificados ${ }^{22}$ pode ter influenciado na menor prevalência de sintomas depressivos neste estudo em contraste aos demais. O trabalho realizado no Nordeste avaliou idosos de uma cidade pequena, com parcos recursos sociais de modo geral23. Da mesma forma, o estudo de Bambuí se deu em uma comunidade pobre, com 43\% da população economicamente ativa ganhando menos da metade de um salário-mínimo ${ }^{4}$. No presente trabalho, quase $80 \%$ dos respondentes afirmaram ter renda familiar inferior a 3 salários-mínimos, o que pode ter corroborado a alta prevalência de sintomas depressivos encontrados.

A associação entre sintomas depressivos e dependência funcional (seja para atividades básicas ou instrumentais) está bem estabelecida na literatura científica ${ }^{10,24}$, e estudos apontam para uma relação bidirecional de causa e efeito entre sintomas depressivos e declínio funcional ${ }^{9,11,12}$. No presente estudo, foi verificada a associação entre sintomas depressivos e dependência para as AIVD, porém o mesmo não ocorreu para ABVD. O conflito entre os resultados deste estudo com os achados da literatura pode ter origem, em especial, no número de pessoas entrevistadas. Enquanto no trabalho em questão foram avaliados 258 idosos, nas pesquisas em que essa associação está bem descrita o número de indivíduos estudados nunca foi inferior a 3 mil. A baixa prevalência de dependência funcional para ABVD $(3,1 \%)$ no presente estudo, dentro de um universo restrito, teria prejudicado tal avaliação. No entanto, a associação entre sintomas depressivos e dependência para AIVD, porém sem associação com dependência para $A B V D$, também é descrita em trabalho realizado com número semelhante de idosos moradores de uma comunidade no nordeste do Brasil23.

Duas das mais importantes referências bibliográficas em saúde mental, o DSM-IV e a CID-10, colocam o sono perturbado como importante aspecto a ser investigado para chegar ao diagnóstico de depressão ${ }^{19,25}$. Entretanto, é bastante conhecida a presença de sono dificultado entre idosos. Pro- blemas com o sono ocorrem em mais da metade das pessoas com 65 anos ou mais, e estima-se que a insônia afete aproximadamente um terço dos idosos nos Estados Uni$\operatorname{dos}^{26}$. A alta prevalência de dificuldades com o sono entre idosos dificulta a avaliação diagnóstica de possíveis doenças associadas. Porém, não existe justificativa plausível para a não investigação da presença desses distúrbios nesses indivíduos, já que as queixas de insônia aumentam cerca de 5\% ao ano na população idosa em geral, porém aumentam apenas em torno de 1\% nos indivíduos mais sadios com 65 anos ou mais ${ }^{26}$. Ademais, os distúrbios do sono também já foram descritos na literatura como fator de risco para depressão?. O presente trabalho apresentou como significativamente associados sintomas depressivos e dificuldade para dormir, com força de associação de cerca de duas vezes, justificando uma investigação mais minuciosa das consequências do sono perturbado no humor de idosos não institucionalizados. Sono prejudicado em idosos muitas vezes pode estar relacionado à dor, em especial naqueles afetados por doenças crônicas com quadro álgico importante, como artrite e câncer ${ }^{26}$. Não foi avaliada no presente estudo variável sobre esse fator nos indivíduos entrevistados, mas talvez seja interessante em pesquisas vindouras a incorporação de uma escala de dor a fim de estabelecer a real influência dela no sono de pessoas acima de 60 anos.

Luto e piores condições de saúde, dois fatores que poderiam ser esperados como associados aos sintomas depressivos nesta pesquisa, não foram detectados como tal. Uma das possíveis causas desse achado pode residir no próprio instrumento de coleta, o questionário BOAS. Nele, o entrevistado é questionado sobre a perda de parente ou companheiro nos últimos meses. Tal pergunta não define exatamente o grau de intimidade entre o entrevistado e esse parente perdido, tampouco estabelece um número claro de meses da morte desse parente, ou seja, a subjetividade da questão pode ter interferido para uma definição não muito precisa de sentimentos de luto. Da mesma maneira pode ter ocorrido com relação às condições de saúde. No instrumento BOAS, é perguntado ao entrevistado se ele possui algum problema de saúde, e não exatamente se ele interpreta suas condições de saúde como precárias. Dessa forma, não é de admirar a alta prevalência de uma resposta afirmativa para essa questão (86\%), uma vez que com o envelhecimento aumenta o risco de se ter pelo menos uma doença crônica ${ }^{13}$. Trabalhos brasileiros que demonstraram associação entre piores condições de saúde e sintomas depressivos utilizaram para mensurar esse item questões sobre a autopercepção de saúde nos idosos avaliados ${ }^{34,23}$.

No presente trabalho, o sexo feminino, outro fator considerado de risco9 ${ }^{9}$ também não foi associado de modo estatisticamente significativo aos sintomas depressivos. Estudo brasileiro realizado no município de São Paulo foi condizente com esse achado da literatura ${ }^{22}$. No entanto, três diferentes estudos conduzidos em distintas regiões do Brasil não en- 
contraram essa associação 3,4,23. Dois desses estudos foram realizados em cidades do interior ${ }^{4,23}$, um deles percorrendo diversas áreas, urbanas e rurais, do estado do Rio Grande do Sul ${ }^{3}$. Tal exposição pode fazer pensar se a relação entre sintomas depressivos em mulheres idosas não se faz mais presente nos grandes centros urbanos, um questionamento que pode até mesmo ser estudado em futuras pesquisas.

A renda familiar não teve associação com sintomas depressivos neste estudo. Isso pode ter ocorrido em virtude da baixa renda familiar relatada pela maioria dos respondentes, que afirmaram receber menos de 3 salários-mínimos em $78,6 \%$ das vezes. Isso pode ajudar a explicar a alta prevalência de sintomas depressivos encontrada nessa população, porém dificulta a averiguação de associações em virtude do pequeno número de indivíduos nos extratos superiores de faixa de remuneração. Durante a análise estatística deste trabalho, vários modelos foram tentados para essa variável, no intuito de deixar grupos de renda maiores, porém em nenhuma das formas estudadas se conseguiu encontrar associação significativa.

Outra causa plausível para a não associação dos fatores luto e baixa renda com sintomas depressivos seria o número de idosos incluídos no estudo, relativamente pequeno. É bastante provável que estudos com desenho semelhante, porém com maior número de indivíduos estudados, tenham o potencial de dirimir um viés nesse sentido.

O tipo do estudo, de caráter transversal, permitiu verificar a prevalência de depressão e fatores associados na população com bastante clareza. No entanto, o mesmo torna-se limitado com relação a se aprofundar sobre associações verificadas, uma vez que não podem ser estabelecidas relações de causa e efeito entre as condições de saúde encontradas e possíveis exposições. Desta feita, não se pretende no trabalho em questão definir a sintomatologia depressiva como fator causal de quaisquer que sejam as variáveis associadas.

Com relação à validade externa, não é pretensão do trabalho que os resultados aqui encontrados possam ser considerados como generalizáveis às populações idosas das diversas realidades brasileiras, uma vez que os indivíduos em estudo são moradores de uma comunidade bastante específica do município de Montes Claros, MG, e não uma amostra representativa dele. Outros estudos brasileiros também têm seus resultados com capacidade de generalização limitada, uma vez que, da mesma forma, foram realizados em áreas geográficas bastante específicas $4,22,23,27$.

A contribuição deste trabalho é, na verdade, em criar ambiente de reflexão sobre a importância da avaliação dos sintomas depressivos em idosos na atenção primária, bem como de fatores presentes concomitantemente a esses sintomas e que possam chamar a atenção para a presença deles, bem como demonstrar a plausibilidade de sua realização no dia a dia das ESF no Brasil. Metanálise recente de 17 estudos na atenção primária concluiu que a EDG-15 ajudou a identificar 4 casos a mais de depressão e descartar 4 casos de pacientes sem depressão a cada 100 atendimentos comparativamente à habilidade geral diagnóstica de médicos de família em detectar depressão em idosos, demandando, no entanto, cerca de 3 a 4 minutos a mais por consulta para sua aplicação ${ }^{28}$, ou seja, não se trata de um teste que toma muito tempo para ser aplicado, uma preocupação constante dos médicos que atendem nas ESF ante a demanda sempre grande por atendimentos nessas unidades.

O tempo gasto inicialmente com o treinamento e posteriormente na aplicação dos instrumentos utilizados nessa avaliação justifica-se, uma vez que os benefícios são evidentes. Estudo com idosos acompanhados na atenção primária demonstrou que aqueles em tratamento para depressão não demonstravam escores aumentados em uma escala validada, indicando o bom prognóstico da terapêutica ${ }^{29}$. Em estudo longitudinal realizado com idosos residindo na comunidade, foram avaliados os fatores associados a uma melhora da percepção da qualidade de vida relacionada à saúde daqueles com depressão. Após 12 meses da avaliação inicial, os fatores que tiveram impacto significativo nessa percepção foram o tratamento para a depressão, o manejo das doenças físicas e a melhora das AIVD ${ }^{30}$.

Dessa forma, qualquer equipe de ESF no Brasil pode, com a devida organização, fazer trabalho de rastreamento semelhante em suas comunidades, de forma sistemática, e, com base nas informações obtidas, realizar ações no sentido de ofertar tratamento e cuidados melhores para a população idosa.

Ante achados aqui discutidos, novas investigações sobre o tema devem continuar a ser feitas. Novos estudos, em especial de caráter longitudinal e com número maior de indivíduos, podem elucidar melhor a relação de causalidade dos fatores associados, bem como benefícios de intervenções realizadas em pacientes expostos a esses fatores ao longo do tempo.

\section{CONCLUSÕES}

O presente trabalho identificou alta prevalência $(20,9 \%)$ de sintomas depressivos na população idosa investigada. Esses sintomas estiveram significativamente associados a dependência funcional para AIVD e dificuldade para dormir.

Com número cada vez mais significativo de idosos, é essencial que os serviços de saúde do Brasil, em especial os de atenção primária, estejam preparados para acolher, avaliar e tomar as medidas preventivas e curativas pertinentes a essa faixa etária. Investigar sintomas depressivos e avaliar a capacidade funcional e outros fatores associados, como o sono, descrito neste estudo, é de suma importância para a manutenção ou melhoria da qualidade de vida de pacientes idosos em suas comunidades. A divulgação dessas informações deve ser ampla, pois muitas investigações provavelmente não são realizadas até hoje em virtude da crença comum, mesmo entre a comunidade médica, de que sintomas depressivos e difi- 
culdade para dormir são constitucionais do idoso. Tal investigação pode ser feita sem a necessidade de grandes recursos, requerendo para tanto apenas a organização do serviço e o treinamento para a aplicação das escalas a serem utilizadas.

\section{REFERÊNCIAS}

1. Vargas HS. A depressão no idoso: fundamentos. São Paulo: BYK; 1992.

2. Snowdon J. How high is the prevalence of depression in old age? Rev Bras Psiquiatr. 2002;24(Sup I):42-7.

3. Blay SL, Andreoli SB, Fillenbaum GG, Gastal FL. Depression morbidity in later life: prevalence and correlates in a developing country. Am J Geriatr Psychiatry. 2007;15(9):790-9.

4. Costa E, Barreto SM, Uchoa E, Firmo JOA, Lima-Costa MF, Prince M. Prevalence of International Classification of Diseases, $10^{\text {th }}$ Revision common mental disorders in the elderly in a Brazilian community: the Bambuí health aging study. Am J Geriatr Psychiatry. 2007;15(1):17-27.

5. Gazalle FK, Hallal PC, Lima MS. Depressão na população idosa: os médicos estão investigando? Rev Bras Psiquiatr. 2004;26(3):145-9.

6. Ruegg RG, Zisook S, Swerdlow NR. Depression in the aged. An overview. Psychiatr Clin North Am. 1988;11(1):83-99.

7. Snowdon J. The prevalence of depression in old age. Int J Geriatr Psychiatry. 1990;5:141-4.

8. Katona CLE. Depression and physicall illness in old age. In: Katona CLE. Depression in old age. West Sussex: Wiley; 1994, p. 63-78.

9. Cole MG, Dendukuri N. Risk factors for depression among elderly community subjects: a systematic review and meta-analysis. Am J Psychiatry. 2003;160:1147-56.

10. Barrantes-Monge M, García-Mayo EJ, Gutiérrez-Robledo LM, Miguel-Jaimes A. Dependencia funcional y enfermedades crónicas en ancianos mexicanos. Salud Publica Mex. 2007;49(Supl 4):S459-66.

11. Ávila-Funes JA, Melano-Carranza E, Payette H, Amieva H. Síntomas depresivos como factor de riesgo de dependencia en adultos mayores. Salud Publica Mex. 2007;49:367-75.

12. Schillerstrom JE, Royall DR, Palmer RF. Depression, disability and intermediate pathways: a review of longitudinal studies in elders. J Geriatr Psychiatry Neurol. 2008;21(3):183-97.

13. World Health Organization. Towards age-friendly primary health care. Geneva; 2004.

14. Brucki SMD, Nitrini R, Caramelli P, Bertolucci PHF, Okamoto IH. Sugestões para 0 uso do Mini Exame do Estado Mental no Brasil. Arq Neuropsiquiatr. 2003;61(3B):777-81.
15. Veras RP, Souza CAM, Cardoso RF, Milioli R, Silva SD. Pesquisando populações idosas A importância do instrumento e o treinamento de equipe: uma contribuição metodológica. Rev Saude Publica. 1988;22:513-8.

16. Almeida OP, Almeida SA. Confiabilidade da versão brasileira da escala de depressão em geriatria (GDS) versão reduzida. Arq Neuropsiquiatr. 1999;57(2B):421-6.

17. Katz S, Ford AB, Moskowitz RW, Jackson BA, Jaffe MW. Studies of illness in the aged. The index of ADL: a standardized measure of biological and psychosocial function. JAMA. 1963;185(12):914-9.

18. Pfeffer RI, Kurosaki TT, Harrah CH Jr, Chance JM, Filos S. Measurement of functional activities in older adults in the community. J Gerontol. 1982;37(3):323-9.

19. American Psychiatric Association. Manual diagnóstico e estatístico de transtornos mentais - 4a edição (DSM-IV). Porto Alegre: Artes Médicas; 1994.

20. Hosmer DW, Lemeshow S. Applied logistic regression. New York: John Wiley; 1989.

21. Instituto Brasileiro de Geografia e Estatística. Perfil dos idosos responsáveis pelos domicílios no Brasil: 2000. Rio de Janeiro: IBGE; 2002.

22. Barcelos-Ferreira R, Pinto JA Jr, Nakano EY, Steffens DC, Litvoc J, Bottino CM. Clinically significant depressive symptoms and associated factors in community elderly subjects from Sao Paulo, Brazil. Am J Geriatr Psychiatry. 2009;17(7):582-90.

23. Maciel ACC, Guerra RO. Prevalência e fatores associados à sintomatologia depressiva em idosos residentes no Nordeste do Brasil. J Bras Psiquiatr. 2006;55(1):26-33.

24. Blazer D, Burchett B, Service C, George LK. The association of age and depression among the elderly: an epidemiologic exploration. J Gerontol. 1991;46(6):210-5.

25. Organização Mundial da Saúde. CID-10: Classificação de Transtornos Mentais e de Comportamento. Descrições clínicas e diretrizes diagnósticas. Porto Alegre: Artes Médicas; 1993.

26. Kryger M, Monjan A, Bliwise D, Ancoli-Israel S. Sleep, health, and aging. Geriatrics. 2004:59:24-30.

27. Costa EC, Nakatani AYK, Bachion MM. Capacidade de idosos da comunidade para desenvolver Atividades de Vida Diária e Atividades Instrumentais de Vida Diária. Acta Paul Enferm. 2006;19(1):43-35.

28. Mitchell AJ, Bird V, Rizzo M, Meader N. Diagnostic validity and added value of the geriatric depression scale for depression in primary care: a meta-analysis of GDS30 and GDS15. J Affect Disord. 2010;125:10-7. Epub 2009 0ct 2.

29. Magnil M, Gunnarsson R, Björkstedt K, Björkelund C. Prevalence of depressive symptoms and associated factors in elderly primary care patients: a descriptive study. Prim Care Companion J Clin Psychiatry. 2008;10(6):462-8.

30. Chan SW, Chiu HFK, Chien WT, Goggins W, Thompson D, Hong B. Predictors of change in health-related quality of life among older people with depression: a longitudinal study. Int Psychogeriatr. 2009;21(6):1171-9. 